\title{
NOTE SUR LA COMPOSITION EN MATIÈRES AZOTÉES DES FROMAGES AFFINÉS DE CAMEMBERT SAINT-PAULIN ET GRUYĖRE DE COMTÉ (1)
}

\author{
par J. LENOIR \\ avec la collaboration technique de Antoinette JoUAN \\ Laboratoire de Technologie, \\ École nationale supérieure agronomique, Grignon (Seine-et-Oise)
}

Les fromages de Camembert, Saint-Paulin et Gruyère de Comté occupent sur le marché français des produits laitiers une place importante (2). Les travaux portant sur divers aspects de leur fabrication sont nombreux; par contre, les données relatives à la protéolyse qui intervient au cours de leur maturation sont rares.

Lindet et Ammann (1904) ont suivi la solubilisation progressive de l'azote et la formation de l'ammoniaque sur ces trois types de fromages. Ils ont noté des différences très marquées dans la composition des fromages mûrs; ainsi les teneurs en azote soluble et en azote ammoniacal (p. 100 de l'azote total) seraient respectivement 98,3 et 12,2 pour le Camembert, 20,7 et 0,5 pour le PortSalut, 24 et 1,1 pour le Gruyère. Le matériel étudié par ces auteurs était sans doute assez différent des fabrications actuelles et leurs résultats ne semblent intéresser qu'un petit nombre d'échantillons de chaque type. Aussi ne peut-on considérer ces résultats comme réellement représentatifs. D'ailleurs, des travaux postérieurs (PIEN et MAURICE, 1937) signalent des chiffres qui ne s'accordent guère avec les précédents et, récemment (LENOIR, 1962), nous avons noté sur des Camemberts affinés des teneurs en azote soluble singulièrement moins élevées que celles obtenues par Lindet et Ammann. Il nous a done paru intéressant de préciser la composition en matière azotées des fromages mûrs et de rechercher si cette composition était assez constante pour caractériser le type de fromage.

\section{PROTOCOLE EXPERIMENTAL}

\section{A. - Prélèvement des échantillons}

Dix échantillons de chaque type de fromage, dont l'apparence indiquait un degré optimal de maturité et qui présentaient des qualités organoleptiques jugées satisfaisantes, ont été prélevés

(1) Ann. Technol. Agric., 1963, 12 (1) 51.

(2) Sur une production fromagère voisine de 450000 tonnes en 1960 le Camembert représente 90000 tonnes, le Saint-Paulin près de 40000 tonnes et le Gruyère de Comté 30000 tonnes (Jarrousse $H$. et Mocquot $G$., communication personnelle). 
dans le commerce. Les fromages de Camembert, tous originaires de Normandie, provenaient de plusieurs fromageries et étaient fabriqués à partir de lait cru. Les fromages de Saint-Paulin, commercialisés sous différentes marques ou appellations, étaient préparés avec du lait pasteurisé. Les fromages de Gruyère de Comté répondaient aux exigences de la législation concernant ce produit dont on sait qu'il bénéficie d'une appellation d'origine.

\section{B. - Méthodes d'analyses}

Après mise en solution dans le citrate de soude (KNUDSEN et Overby, 1942 ; Mogensen, 1947) d'une partie aliquote du fromage, le fractionnement des matières azotées a été effectué selon la technique antérieurement décrite (LENorr, 1962). Outre la détermination des formes suivantes : azote total (NT), azote soluble total (NST), azote non protéique (NPN), azote formol (NF) et azote ammoniacal $\left(\mathrm{N} \mathrm{NH}_{3}\right.$ ), l'analyse a comporté une détermination de l'azote soluble dans l'acide phosphotungstique à 2 p. 100 en milieu sulfurique $2 \mathrm{~N}$ (NPT). Cette dernière forme de l'azote peut être assimilée aux composés de faible poids moléculaire, oligopeptides et acides aminés neutres et acides (MogENSEN, 1947).

\section{RÉSULTATS}

Les résultats analytiques sont groupés dans les tableaux 1, 2 et 3. Ces tableaux indiquent les teneurs moyennes des formes solubles de l'azote, les caractéristiques de dispersion de chaque série d'échantillons, écart-type et coefficient de variation, et les valeurs probables des moyennes calculées selon la méthode STUDent-Fisher. Ces diverses données montrent qu'il existe, pour chaque type de fromage, une certaine constance dans les teneurs en azote soluble, constance plus marquée toutefois pour les formes NST et NPN que pour les fractions $\mathrm{NPT}, \mathrm{NF}$ et $\mathrm{NNH}_{3}$. On remarque, en outre, que la composition des fromages de Camembert et de Gruyère de Comté varie sensiblement moins d'un échantillon à l'autre que celle des fromages de Saint-Paulin. Cette observation peut, au premier abord, paraître curieuse. En effet, le Saint-Paulin est préparé avec du lait pasteurisé et, la coagulation, l'égouttage et la maturation du caillé sont en grande partie l'œuvre de levains lactiques. Il devrait logiquement s'ensuivre une certaine uniformité des caractères analytiques, uniformité de toute façon plus marquée que pour le Camembert normand, fabriqué à partir de lait cru. Cette dispersion relative des résultats concernant le SaintPaulin est vraisemblablement liée à la diversité des caractères des 
levains lactiques employés et à l'intervention, dans le processus de maturation de certains échantillons, de microorganismes autres que les bactéries lactiques, microcoques et levures notamment (Veisseyre, Lenoir et Marceron).

Les résultats relatifs aux fromages de Camembert (tableau 1) montrent que l'azote total est formé de 31 à 34 p. 100 d'azote soluble, 7,5 à 10 p. 100 d'azote soluble dans l'acide phosphotungstique et 6,5 à 9 p. 100 d'azote ammoniacal. Ces résultats confirment certaines données publiées antérieurement (JACQUET et LENOIR, 1954 ; LenoIr, 1962) et montrent que la dégradation de la caséine est nettement moins forte que ne l'indiquaient les travaux anciens (Lindet et AmmanN, 1904 ; Bosworth, 1907). Les caractères analytiques des fromages de Saint-Paulin (tableau II) révèlent un taux de protéolyse peu prononcé; l'azote soluble ne représente que 15,5 à 21,5 p. 100 de l'azote total, l'azote soluble dans l'acide phosphotungstique 2,5 à 5,5 p. 100 et l'azote ammoniacal 0,3 à 2 p. 100. Ces résultats concordent avec ceux précédemment cités de Lindet et Ammann (1904). Quant au Gruyère de Comté (tableau III), s'il est assez proche du Camembert par ses teneurs en

\section{TABLEAU I}

FORMES SOLUBLES DE L'AZOTE DU CAMEMBERT AFFINÉ

\begin{tabular}{|c|c|c|c|c|}
\hline Formes de l'azote & $\begin{array}{c}\text { Moyenne M } \\
(\% \mathrm{NT})\end{array}$ & Ecart-type & $\begin{array}{l}\text { Coefficient } \\
\text { devariation }\end{array}$ & $\begin{array}{c}\text { M probable } \\
\text { t }(95)(\% \text { NT) }\end{array}$ \\
\hline Azote soluble total $\ldots \ldots$ & 32,46 & 1,65 & 0,050 & $31,28-33,64$ \\
\hline Azote non protéique $\ldots$. & 20,70 & 1,91 & 0,092 & $19,34-22,06$ \\
\hline Azote phosphotungstique. & 8,69 & 1,11 & 0,128 & $7,84-9,54$ \\
\hline Azote formol $\ldots \ldots \ldots \ldots$ & 11,09 & 1,62 & 0,146 & $9,94-12,24$ \\
\hline Azote ammoniacal $\ldots . .$. & 7,73 & 1,29 & 0,167 & $6,81-8,65$ \\
\hline
\end{tabular}

TABLEAU II

FORMES SOLUBLES DE L'AZOTE DU SAINT-PAULIN AFFINÉ

\begin{tabular}{|c|c|c|c|c|}
\hline Formes de l'azote & $\begin{array}{c}\text { Moyenne M } \\
(\% \mathrm{NT})\end{array}$ & Ecart-type & $\begin{array}{c}\text { Coefficient } \\
\text { devariation }\end{array}$ & $\begin{array}{c}\text { M probable } \\
t(95)(\% \mathrm{NT})\end{array}$ \\
\hline Azote soluble total $\ldots \ldots$. & 18,41 & 2,97 & 0,161 & $16,29-20,53$ \\
\hline Azote non protéique $\ldots .$. & 10,03 & 2,04 & 0,204 & $8,57-11,49$ \\
\hline Azote phosphotungstique. & 4,05 & 1,46 & 0,365 & $3,01-5,09$ \\
\hline Azote formol $\ldots \ldots \ldots \ldots$ & 3,93 & 1,29 & 0,331 & $3,01-4,85$ \\
\hline Azote ammoniacal . . . . . & 1,03 & 0,74 & 0,740 & $0,50-1,56$ \\
\hline
\end{tabular}




\section{TABLEAU III}

FORMES SOLUBLES DE L'AZOTE DU GRUYÈRE DE GOMTÉ AFFINÊ

\begin{tabular}{c|c|c|c|c}
\hline \hline \multicolumn{1}{c|}{ Formes de l'azote } & $\begin{array}{c}\text { Moyenne } \mathbf{M} \\
(\% \mathrm{NT})\end{array}$ & Ecart-type & $\begin{array}{c}\text { Coefficient } \\
\text { devariation }\end{array}$ & $\begin{array}{c}\text { M probable } \\
t(95)(\% \text { NT) }\end{array}$ \\
\cline { 1 - 4 } Azote soluble total ...... & 30,23 & 2,40 & 0,079 & $28,52-31,94$ \\
Azote non protéique ..... & 23,97 & 2,61 & 0,109 & $22,10-25,84$ \\
Azote phosphotungstique. & 16,42 & 1,92 & 0,117 & $15,05-17,79$ \\
Azote formol ........... & 14,23 & 1,58 & 0,111 & $13,10-15,36$ \\
Azote ammoniacal ....... & 3,81 & 0,86 & 0,230 & $3,20-4,42$ \\
\hline \hline
\end{tabular}

azote soluble ( 28 à 33 p. 100), en azote non protéique, voire même en azote formol, il s'en distingue par les proportions d'azote soluble dans l'acide phosphotungstique $(14,5$ à 18,5 p. 100) et d'azote ammoniacal ( 3 à 4,7 p. 100).

Les différences de composition entre ces trois types de fromage apparaissent plus clairement si l'on envisage les diverses fractions azotées (tableau IV). On constate notamment que le Saint-Paulin, qui présente la teneur la plus élevée en $\mathrm{N}$ caséine (plus de 80 p. 100, alors que le Camembert et le Gruyère de Comté en contiennent un peu moins de 70 p. 100), est aussi le fromage dont l'azote soluble a subi la dégradation la moins profonde. L'azote soluble du Camembert est déjà plus riche en fractions simples; il contient en effet moins d'azote protéose et plus d'azote ammoniacal.

\section{TABLEAU IV}

GOMPOSITION AZOTEE MOYENNE DES FROMAGES DE GAMEMBERT, SAINT-PAULIN ET GRUYĖRE DE COMTÉ

\begin{tabular}{|c|c|c|c|c|c|c|}
\hline \multirow{2}{*}{$\begin{array}{l}\text { Formes de } \\
\text { l'azote (1) }\end{array}$} & \multicolumn{3}{|c|}{$\% \mathrm{NT}$} & \multicolumn{3}{|c|}{$\%$ NST } \\
\hline & $\begin{array}{l}\text { Camem- } \\
\text { bert }\end{array}$ & $\begin{array}{l}\text { Saint } \\
\text { Paulin }\end{array}$ & $\begin{array}{c}\text { Gruyère } \\
\text { de Comté }\end{array}$ & $\begin{array}{l}\text { Camem- } \\
\text { bert }\end{array}$ & $\begin{array}{l}\text { Saint } \\
\text { Paulin }\end{array}$ & $\begin{array}{c}\text { Gruyère } \\
\text { de Comté }\end{array}$ \\
\hline N Caséine & 67,5 & 81,6 & 69,8 & - & - & - \\
\hline N Protéose. & 11,8 & 8,4 & 6,3 & 36,2 & 45,5 & 20,7 \\
\hline N Peptide ..... & 9,6 & 6,1 & 9,7 & 29,6 & 33,1 & 32,2 \\
\hline N Aminé ...... & 3,4 & 2,9 & 10,4 & 10,3 & 15,7 & 34,4 \\
\hline N Ammoniacal & 7,7 & 1,0 & 3,8 & 23,8 & 5,6 & 12,6 \\
\hline
\end{tabular}

(1) $\mathrm{N}$ Caséine $=\mathrm{NT}-\mathrm{NST}$;

$\mathrm{N}$ Protéose $=\mathrm{NST}-\mathrm{NPN}$;

$\mathrm{N}$ Peptide $=\mathrm{NPN}-\mathrm{NF}$;

$\mathrm{N}$ Aminé $=\mathrm{NF}-\mathrm{N} \mathrm{NH}_{3}$. 
Plus simplifié aussi est l'azote du Gruyère de Comté dans lequel l'azote formol représente plus de 45 p. 100 de l'azote soluble et l'azote aminé près de 75 p. 100 de l'azote formol.

\section{DISCUSSION}

Les fromages étudiés, Camembert, Saint-Paulin et Gruyère de Comté, sont effectivement caractérisés par leur composition en matières azotées. Pour un même type de fromage, cette composition varie relativement peu d'un échantillon à l'autre et les teneurs moyennes en diverses formes de l'azote trouvées dans ce travail peuvent être considérées comme représentatives du type de fromage étudié. Des différences significatives entre chacun des types sont, par contre, mises en évidence. Ces différences ne portent pas seulement sur "l'étendue" de la protéolyse concrétisée par les teneurs en azote soluble total, elles portent aussi sur la "qualité " de cette protéolyse exprimée par les taux des diverses fractions azotées solubles : protéoses, peptides, acides aminés et ammoniaque. Parmi les caractères distinctifs particulièrement marquants on peut retenir les teneurs en azote soluble total, en azote aminé et en azote ammoniacal, et le rapport $\mathrm{N}$ aminé/ $\mathrm{N}$ ammoniacal. Le tableau $\mathrm{V}$ résume ces caractères.

\section{TABLEAU $V$}

CARAGTERES DISTINCTIFS DES FROMAGES DE CAMEMBERT, SAINT-PAULIN ET GRUYẼRE DE COMTÉ

\begin{tabular}{|c|c|c|c|}
\hline & Camembert & Saint-Paulin & Gruyère de Comté \\
\hline $\mathrm{N}$ soluble total ${ }^{1} \ldots \ldots \ldots$ & $31-34$ & $16-21$ & $28-32$ \\
\hline $\mathrm{N}$ aminé ${ }^{2} \ldots \ldots \ldots \ldots$ & $9-12$ & $13-18,5$ & $32-37$ \\
\hline $\mathrm{N}_{\text {ammoniacal }}{ }^{2} \ldots \ldots \ldots$ & $21-27$ & $2,7-8,5$ & $10,5-14,5$ \\
\hline $\mathrm{N}$ aminé/N ammoniacal. & $0,35-0,50$ & $2,1-7,5$ & $2,4-3,3$ \\
\hline
\end{tabular}

(1) P. 100 de l'azote total.

(2) P. 100 de l'azote soluble total.

La composition en matières azotées d'un fromage affiné n'est que la traduction de l'activité, au cours de la fabrication et de la maturation, des agents responsables de la dégradation des protéines, la présure et les microorganismes. En ce qui concerne le rôle possible des microorganismes on remarque que les fromages dont la maturation est surtout l'œuvre de bactéries lactiques (SaintPaulin et Gruyère de Comté) contiennent peu d'azote ammoniacal et relativement beaucoup d'azote aminé. Par contre, ceux du type 
Camembert, dont l'affinage est l'œuvre d'une flore complexe au sein de laquelle moisissures, levures et microcoques jouent un rôle dominant présentent un rapport $\mathrm{N}$ aminé/ $\mathrm{N}$ ammoniacal très inférieur à 1. Ces différences peuvent être attribuées à l'activité des désaminases d'origine microbienne, activité qui ne se manifeste nettement que dans les fromages à pâte molle (RAIBAUD et al., 1959). Comme par ailleurs, certaines observations (VEIsserre, Lenotr et Pierre) ont montré que les acides diaminés libres étaient, en pourcentage relatif, moins abondants dans le Camembert que dans le Saint-Paulin ou le Gruyère de Comté, on peut penser que la désamination porte principalement sur ces acides diaminés. On note également que le Saint-Paulin et le Gruyère de Comté, bien que tous deux à maturation lactique dominante, se distinguent par la complexité de leurs matières azotées solubles. Les composés de faible poids moléculaire, oligopeptides et acides aminés, sont en effet singulièrement plus abondants dans le Gruyère de Comté, où ils forment 50 à 58 p. 100 de l'azote soluble, que dans le SaintPaulin, où ils ne représentent que 16 à 28 p. 100 . Une telle différence, qui résulte des activités respectives des protéinases et des peptidases microbiennes, ne peut vraisemblablement s'expliquer par la seule durée de l'affinage. L'intervention dans la maturation du Gruyère de Comté des lactobacilles, dont on connaît l'activité protéolytique, peut contribuer à expliquer cette différence. D'autre part, les levures, présentes dans certains fromages de Saint-Paulin, sont aptes à libérer une quantité notable d'azote soluble sous la forme de composés de poids moléculaire élevé (VEISSEYRE, LENoIR et Marceron); elles peuvent donc intervenir aussi dans le phénomène.

Ces observations n'intéressent qu'un aspect de la protéolyse qui se produit au cours de la maturation. Une étude de la distribution de l'azote des fromages ne peut ignorer ni les acides aminés libres, ni les produits de décarboxylation et de désamination des acides aminés. En outre, pour interpréter une telle étude, il faut aussi connaître, de façon précise, la flore microbienne du type du fromage et son comportement spécifique vis-à-vis des matières azotées.

\section{RÉSUMÉ}

Sur dix échantillons de fromages affinés de chacun des types Camembert, Saint-Paulin et Gruyère de Comté, les teneurs en diverses formes d'azote soluble, azote soluble total, azote non protéique, azote soluble dans l'acide phosphotungstique, azote aminé et azote ammoniacal, ont été déterminées.

La composition en matières azotées de chaque type de fromage 
présente une certaine constance. Par contre des différences significatives entre chacun des types sont notées. Ainsi, l'azote soluble total représente dans le Camembert 31 à 34 p. 100 de l'azote total, dans le Saint-Paulin 16 à 21 p. 100, dans le Gruyère de Comté 28 à 32 p. 100. La composition de cet azote soluble est elle-même sensiblement différente d'un type de fromage à l'autre. Les pourcentages respectifs en azote aminé et en azote ammoniacal sont de 9 à 12 et 21 à 27 dans le Camembert, 13 à 18,5 et 2,7 à 8,5 dans le Saint-Paulin, 32 à 37 et 10,5 à 14,5 dans le Gruyère de Comté (pourcentages rapportés à l'azote soluble total).

\section{SUMMARY}

\section{Note on the composition of nitrogen constituents in ripe camembert, saint-Paulin and Gruyère de comté cheeses}

The amounts of the different forms of soluble nitrogen, total soluble nitrogen, non-protein nitrogen, nitrogen soluble in phosphotungstic acid, amino nitrogen and ammoniacal nitrogen were determined in 10 samples of each of the cheeses : Camembert, Saint-Paulin and Gruyère de Comté.

While the nitrogen constituents in each particular cheese variety showed a certain constancy, there were significant differences between varieties. Thus, total soluble nitrogen amounted to 31-34 p. 100 of total nitrogen in Camembert, 16-21 p. 100 in SaintPaulin and 28-32 p. 100 in Gruyère de Comté. The composition of this soluble nitrogen differed appreciably from one variety of cheese to another. Amino nitrogen and ammoniacal nitrogen in Camembert amounted to $9-12$ p. 100 and $21-27$ p. 100 respectively, while the comparable figures for Saint-Paulin were $13-18,5$ p. 100 and 2.7-8.5 p. 100 and for Gruyère de Comté $32-37$ p. 100 and 10.5-14.5 p. 100 (figures shown as percentages of total soluble nitrogen).

\section{RÉFÉRENCES BIBLIOGRAPHIQUES}

Bosworth A. W., 1907. D'après Hammer B. W., 1948, Dairy Bacteriology 550 , Wiley and Sons, New York.

JACQUET J., Lenoir J., 1954. Sur l'affinage des fromages à pâte molle du type Camembert C. R. Acad. Sci., 238, 2201-2203.

Knudsen S., Overby A. J., 1942. Kong. Vet. og Land. Aarskr., Copenhague. Lenorr J., 1962. Sur la dégradation des protides au cours de la maturation du Camembert. C. R. Acad. gr., 48, 160-169.

Lindet L., Ammann L., 1904. D'après Lindet L., 1907. Le lait, la crème, le beurre, les tromages, 280, Gauthier-Villars, Paris. 
Mogensen M. T. S., 1947. Appréciation du degré de protéolyse du fromage en prenant plus particulièrement en considération la formol titration (en danois). Meddelande fran statens Mejeriforsök, $\mathrm{n}^{\circ} 21$.

Pien J., Maurice G., 1937. Modifications dans la composition des fromages au cours de leur conservation prolongée, Le Lait, 17, 1040-1046.

Raibaud P., Herren R., Mocquot G., Kosikowski F. V., 1959. Mise en évidence dans certains fromages de systèmes enzymatiques actifs sur la tyrosine. Ann. Technol, agric., 8, 117-129,

Veisseyre R., Lenoir J., Marceron J. F. (Données non publiées).

Veisseyre R., Lenoir J., Pierre M. (Données non publiées).

\title{
L'EMPLOI DES PLASTIQUES POUR L'EMBALLAG DES PRODUITS LAITIERS
}

\author{
par \\ G. GENIN \\ Ingénieur E.P.C.I.
}

Depuis déjà plusieurs années, l'utilisation des plastiques s'est généralisée dans l'emballage et le conditionnement des produits alimentaires et en particulier des produits laitiers. L'emploi des matières plastiques dans l'emballage répond à des buts différents, soit simplement emballer les produits pour les conserver au frais et propres dans une pellicule se conservant elle-même convenablement, soit réaliser un véritable emballage étanche en vue par exemple de conserver des aliments congelés (dans ce cas la feuille d'emballage doit permettre ou non la "respiration du contenu»), soit assurer l'emballage de produits qui doivent ensuite, dans cet état, subir une cuisson, soit assurer la conservation et le conditionnement de produits liquides, soit enfin assurer le stockage de quantités importantes de produits dans de grands entrepôts.

On voit que les problèmes posés sont nombreux et il n'est donc pas étonnant que les solutions proposées soient différentes, en particulier depuis que l'industrie des produits alimentaires a à sa disposition une grande variété de matériaux plastiques. Dans cette étude, nous nous contenterons donc de décrire les pellicules plastiques utilisées dans l'emballage, énoncer leurs propriétés et leurs principales caractéristiques, quitte à revenir ultérieurement sur des formes plus particulières d'emploi des matériaux plastiques disponibles. 